\title{
Adding evidence to the role of NEUROG1 in congenital cranial dysinnervation disorders
}

\author{
Juliette Dupont $^{1}$ (1) | José Pedro Vieira ${ }^{2}$ | Ana Lisa Taylor Tavares ${ }^{3,4}$ | \\ Carla Ribeiro Conceição ${ }^{5}$ | Suliman Khan $^{6}$ | Aida Maria Bertoli-Avella ${ }^{6}$ | \\ Ana Berta Sousa ${ }^{1,7}$
}

\author{
${ }^{1}$ Genetics Department, Hospital de Santa \\ Maria, Centro Hospitalar Universitário de \\ Lisboa Norte, Lisbon, Portugal \\ ${ }^{2}$ Neurology Department, Hospital de Dona \\ Estefânia, Centro Hospitalar Universitário de \\ Lisboa Central, Lisbon, Portugal \\ ${ }^{3}$ East Anglian Medical Genetics Service, \\ Cambridge University Hospitals NHS \\ Foundation Trust, Cambridge, UK \\ ${ }^{4}$ Department of Health, Genomics England, \\ Queen Mary University of London, \\ Charterhouse Square, London, UK \\ ${ }^{5}$ Neuroradiology Department, Hospital de \\ Dona Estefânia, Centro Hospitalar \\ Universitário de Lisboa Central, Lisbon, \\ Portugal \\ ${ }^{6}$ Research Data Analysis, CENTOGENE AG, \\ Rostock, Germany \\ ${ }^{7}$ Laboratório de Imunologia Básica, Faculdade \\ de Medicina da Universidade de Lisboa, \\ Lisbon, Portugal

\section{Correspondence} \\ Juliette Dupont, Genetics Department, \\ Hospital de Santa Maria, Centro Hospitalar \\ Universitário de Lisboa Norte, Lisbon, \\ Portugal. \\ Email: juliette.dupont@chln.min-saude.pt and \\ jdupontg@gmail.com
}

\begin{abstract}
Congenital cranial dysinnervation disorders (CCDDs) are a heterogeneous group of neurodevelopmental phenotypes caused by a primary disturbance of innervation due to deficient, absent, or misguided cranial nerves. Although some CCDDs genes are known, several clinical phenotypes and their aetiologies remain to be elucidated. We describe a 12-year-old boy with hypotonia, developmental delay, sensorineural hearing loss, and keratoconjunctivitis due to lack of corneal reflex. He had a long expressionless face, severe oromotor dysfunction, bilateral agenesis/severe hypoplasia of the VIII nerve with marked atresia of the internal auditory canals and cochlear labyrinth malformation. Trio-exome sequencing identified a homozygous loss of function variant in the NEUROG1 gene (NM_006161.2: c.202G > T, p.Glu68*). NEUROG1 is considered a causal candidate for CCDDs based on (i) the previous report of a patient with a homozygous gene deletion and developmental delay, deafness due to absent bilateral VIII nerves, and severe oromotor dysfunction; (ii) a second patient with a homozygous NEUROG1 missense variant and corneal opacity, absent corneal reflex and intellectual disability; and (iii) the knockout mouse model phenotype which highly resembles the disorder observed in humans. Our findings support the growing compelling evidence that loss of NEUROG1 leads to a very distinctive disorder of cranial nerves development.
\end{abstract}

\section{KEYWORDS}

aplasia/hypoplasia of sensory cranial ganglia, congenital cranial dysinnervation disorder, NEUROG1, oromotor dysfunction, sensorineural deafness, trigeminal nerve aplasia/hypoplasia, vestibulo-cochlear nerve aplasia/hypoplasia

\section{1 | INTRODUCTION}

The abnormal development of the cranial nerves can have a genetic basis and result in several phenotypes including recessive hearing loss and congenital cranial dysinnervation disorders (CCDDs), such as Moebius syndrome, Duane syndrome, Marcus Gunn jaw-winking syndrome, congenital ptosis, and congenital fibrosis of extraocular

Juliette Dupont and José Pedro Vieira contributed equally to this study. muscles. ${ }^{1}$ These disorders are heterogeneous and incompletely understood.

NEUROG1 encodes Ngn1 which is a basic helix-loop-helix transcription factor essential for the formation of the proximal cranial sensory ganglia for cranial nerves $\mathrm{V}$ and VIII. Ngn1 induces neurogenesis and inhibits the differentiation of neural stem cells into astrocytes by two independent mechanisms. Initially expressed in the epithelium of the trigeminal and otic placodes prior to the onset of neuroblast migration, Ngn1 dimerizes with ubiquitous bHLH proteins and triggers 
a cascade of downstream bHLH factors, including NeuroD, MATH3 and NSCL1, leading to neuronal differentiation. In addition, Ngn1 inhibits astrocyte differentiation by sequestering the CREB-binding protein transcription complex away from astrocyte differentiation genes and by inhibiting the activation of STAT transcription factors necessary for gliogenesis. ${ }^{2,3}$

We report a patient with mild intellectual disability, epilepsy, deafness, peripheral sensory neuropathy, loss of facial sensation, abnormal development of sensory cranial nerves $\mathrm{V}$ and VIII, and a homozygous putative loss of function variant in NEUROG1. The patient's phenotype is highly similar to two previously reported cases with homozygous variants in NEUROG1. ${ }^{4,5}$ Although NEUROG1 is not yet associated with a human phenotype in OMIM, our findings support a causal link between NEUROG1 and a CCDD.

\section{2 | PATIENT AND METHODS}

Clinical data was obtained from clinical files and a detailed physical exam was performed. All procedures had the approval of the ethics committee of Hospital de Dona Estefânia, Centro Hospitalar Universitário de Lisboa Central.

Informed written consent was obtained from the patient's parents. All genetic studies were performed for diagnostic purposes.

Clinical data from our patient was compared with published data from two other patients described in the medical literature with homozygous variants in NEUROG1.4,5

The patient and his parents were exome sequenced at CENTOGENE AG (Rostock, Germany), as previously described. ${ }^{6}$ Libraries were sequenced on an Illumina Hiseq 4000 with $150 \mathrm{bp}$ paired end sequencing. Variant calling was performed using GATK. Variants with a phred-scaled quality score $>215^{7}$ and a MAF of $<1 \%$ were retained for analysis. Variants were further filtered based on zygosity and segregation in the family. The database CentoMD $\AA^{8}$ was queried for rare variants (<1\%) in NEUROG1 (14 August 2020) and the resulting data was filtered taking into consideration an autosomal recessive mode of inheritance and the impact of the variant (nonsynonymous).

\section{3 | CLINICAL REPORT}

Our patient is a 12-year-old male, the only child of healthy parents of Portuguese origin with no known consanguinity.

Gestation was uneventful and delivery was by caesarean section at term due to feto-pelvic disproportion. Birth weight was $3395 \mathrm{~g}$ (50th centile), length $51.5 \mathrm{~cm}$ (50-75th centile) and head circumference $34 \mathrm{~cm}$ (25-50th centile). Apgar score was 9/10.

He was globally hypotonic since birth. At 22 days he was admitted to hospital due to hypotonia and feeding difficulties requiring naso-gastric feeds.

He had global developmental delay with no history of regression. $\mathrm{He}$ sat alone at 18 months and walked independently at 3 years. $\mathrm{He}$ never developed expressive language and Brainstem Auditory Evoked Responses at 15 months confirmed profound bilateral sensorineural deafness. He was educated in a school for deaf children and learnt to communicate using sign language.

Ophthalmology review at 18 months was normal; however, at 24 months he had keratoconjuntivitis and lost vision in his right eye. Around the age of five he manifested insomnia, hyperkinesia and selfinjurious behavior (hitting his face and eyes). When he was seven he also presented brief bilateral myoclonic seizures.

At the age of 10 his global IQ (evaluated with appropriate tests for deaf children) was 62 , and he manifested major problems with attention, short term memory and abstract thinking.

On physical examination at 10 years his stature was $129 \mathrm{~cm}$ (10th centile), weight was $24 \mathrm{~kg}$ (third centile), and head circumference $52 \mathrm{~cm}$ (25th centile). He had a long, asymmetric and expressionless face with open mouth (Figure 1). Long palpebral fissures, high arched eyebrows, ocular leucoma in the right eye, and an arched, and narrow palate were noted (Figure 1). Neurological examination revealed deafness, apparent bilateral loss of facial sensation, and absence of corneal reflexes. Ocular motility was normal. Facial movements in emotional expressions were asymmetric with the mouth deviating to the left. Lower cranial nerve examination was normal. Gait was unsteady with no preponderant side. Muscle power and tone were normal. Stretch reflexes were absent and there was a bilateral flexor plantar response.

Extensive neurometabolic investigations, abdominal ultrasound and echocardiogram were all normal. EMG at 5 years was compatible with an axonal sensory neuropathy. Blink reflexes were absent bilaterally. Brain MRI revealed hypoplasia of cranial nerves $\mathrm{V}$ and VIII, marked atresia of the internal auditory canal and hypoplasia of the cochlear labyrinth, bilaterally (Figure 2). The brainstem, cerebellum and cerebral hemispheres were normal. The EEG documented bilateral rolandic spikes with normal background activity.

Array CGH showed no relevant copy number variants. Previous molecular testing of DMPK and SLC52A1/SLC52A2/SLC52A3 were also negative. Trio-exome performed for diagnostic purposes (CENTOGENE AG) did not detect relevant variants in morbid genes. An extended search to include genes not previously associated with human disease identified a novel nonsense homozygous variant in NEUROG1 (NM_006161.2): c.202G > T, p.Glu68*. The gene contains only one exon, and there is only one known NEUROG1 protein isoform. The variant introduces a premature termination codon, leading most likely to a truncated protein. Schematic from BAM files showing the location of the variant and the bHLH protein domain is depicted in Figure S1. Both parents were heterozygous for the NEUROG1 variant thus confirming homozygosity in the index case. There are only a few LoF variants in NEUROG1 reported in gnomAD, none in homozygosity (acessed August 14, 2020). Similarly, a search in CENTOGENE data repositories (PMID: 28116331) did not identify additional individuals with homozygous or combined heterozygous LoF variants in this gene. 


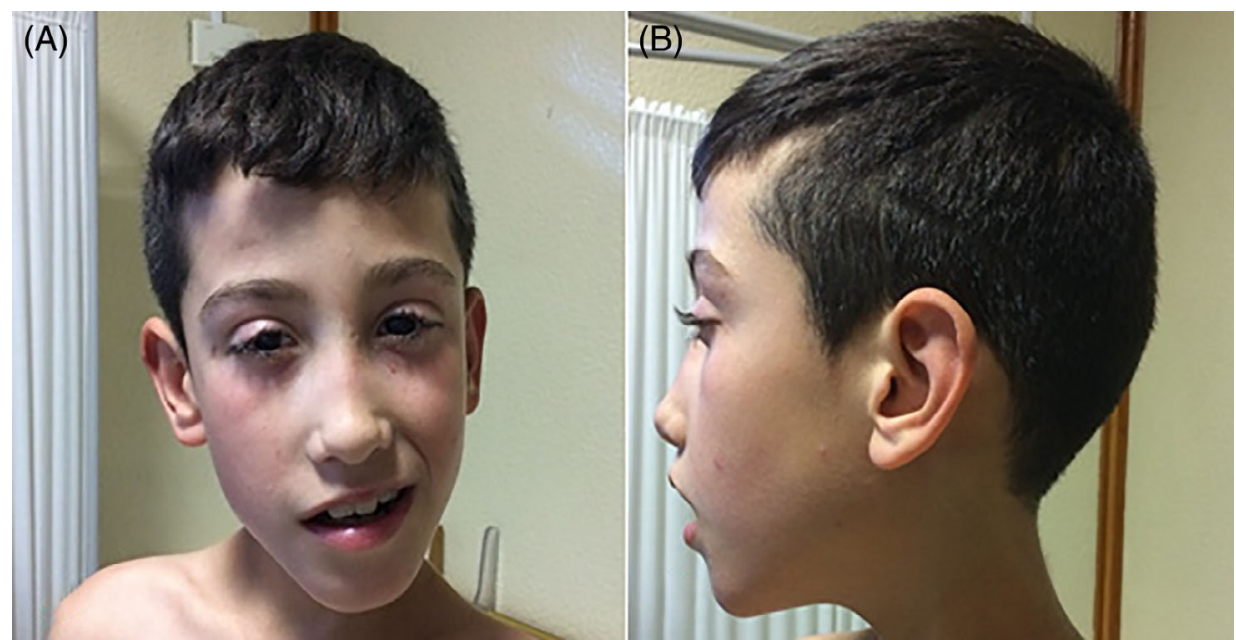

FIGURE 1 Frontal and profile views of our patient with a homozygous nonsense variant in NEUROG1 gene at 10 years: (A) long expressionless face, elongated palpebral fissures, facial asymmetry, deviation of the mouth to the left when smiling with loss of the nasolabial fold on the right-side; (B) inability to close the mouth [Colour figure can be viewed at wileyonlinelibrary.com]
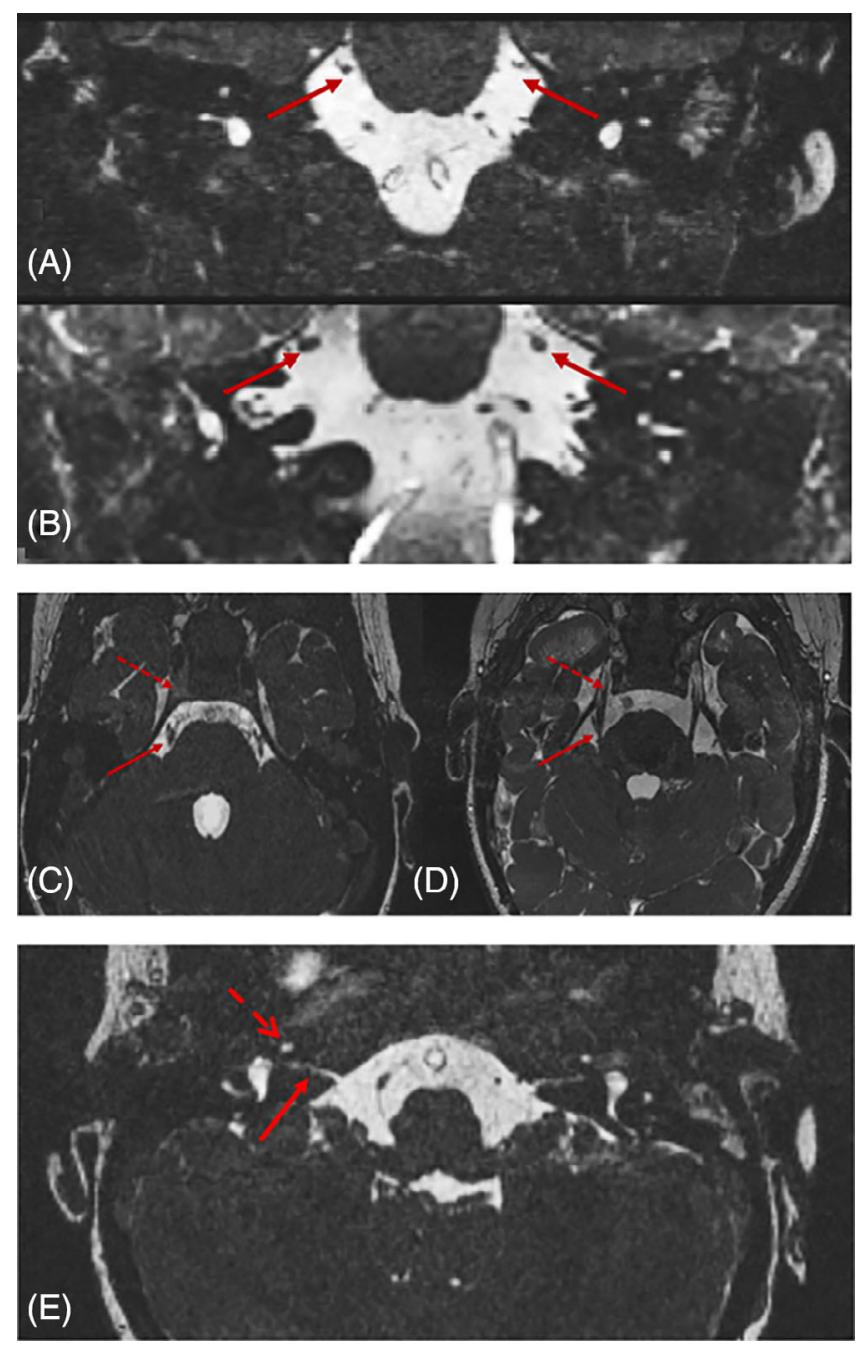

FIGURE 2 T2 weighted brain MRI, Constructive Interference in Steady State (CISS) sequence: (A),(B) Coronal view of the patient's trigeminal nerves $(A)$ showing they are much thinner than in a normal control at the same age (B); (C),(D) Axial view of the patient's trigeminal nerves $(C)$ in comparison with a normal control (D) with the dashed arrows pointing at the trigeminal ganglion; (E) Axial view showing a stenotic internal auditory canal (arrow) and hypoplastic cochlea (dashed arrow) [Colour figure can be viewed at wileyonlinelibrary.com]

\section{4 | DISCUSSION}

Several genes related to neuronal development are currently linked to congenital cranial dysinnervation disorders (PLXND1 and REV3L for Moebius syndrome, MAFB, HOXA1, SALL4 and CHN1 for Duane syndrome, $\mathrm{ROBO} 3$ for horizontal gaze palsy with progressive scoliosis, and PHOX2A, KIF21A, TUBB3 and TUBB2B for congenital fibrosis of extraocular muscles). These disorders are believed to result from a primary disruption of innervation due to the abnormal development of cranial nerves. ${ }^{9-13}$

In the developing CNS the formation of trigeminal and vestibulocochlear nerves requires the activity of NEUROG1., ${ }^{2,3}$ The development of dorsal root ganglion cells is likewise dependent on NEUROG1, which also commits neuronal progenitor cells in the olfactory bulb, cerebral cortex, hippocampus, thalamus, brainstem and cerebellum. ${ }^{14}$ The gene NEUROG1 and protein Ngn1 have been well studied in vertebrate animal models. ${ }^{15,16}$

Ma et al. (1998) generated Ngn1-null mice embryos showing complete absence of trigeminal and otic placode-derived vestibulocochlear ganglia, apparent truncation of cranial nerve $\mathrm{V}$, and loss of expression of Ngn1 downstream bHLH factors, including NeuroD, Math3 and NSCL1, in mutant otic and trigeminal placodes.

Present knowledge about the biology of Ngn1 and the phenotype of the animal model are concordant with the clinical features we report here. Our patient's deafness, hypoplastic cranial nerve VIII, bilateral atresia of the internal auditory canals, and hypoplasia of the cochlear labyrinth match the observations in Neurog1 knock-out mice. ${ }^{15,16}$ Ngn1-null mutants had no vestibulocochlear ganglion, no VIIIth nerve afferent, efferent and autonomic fibres, small abnormal inner ear and cochlear hypoplasia. Our patient's hypoplasia of cranial nerve $\mathrm{V}$, lack of corneal reflexes, and facial anaesthesia were associated with oromotor dysfunction and led to a corneal traumatic lesion. In mice lacking Ngn1 inability to suckle caused early lethality. In the human patients medical resources such as parenteral feeding, nasogastric tube and interventions focusing on strengthening oromotor function helped overcome equivalent feeding difficulties. 
NEUROG1 is involved in neurogenesis in the central nervous system including the neocortex. ${ }^{17}$ Although the intellectual disability present in our patient may be partly ascribed to deafness and sensorial deprivation, and the self-injurious behavior likewise attributed to abnormal sensation in the trigeminal territory, it is likely these features, as well as epilepsy, are manifestations of abnormal cortical development. NEUROG1 promotes sensory neuron differentiation in dorsal root ganglia. ${ }^{18}$ Thus, delayed motor development, and the findings on nerve conduction studies compatible with a subclinical axonal sensory neuropathy in our patient, may also be related to the NEUROG1 gene defect.

In 2013, Schroder et al. ${ }^{4}$ reported one patient with dysmorphic features, mild intellectual disability, deafness, severe hypoplasia of the vestibulo-cochlear nerves, narrow internal auditory canal, hypoplastic cochlea, and oromotor dysfunction. This patient had a homozygous microdeletion in chromosome 5 including all exons and corresponding promoter regions of the NEUROG1, TIFAB and DCNP1 genes. The authors claimed the phenotype in Neurog1 knock-out mice was in agreement with the clinical and radiological findings in their patient. Moreover, they observed a balance disorder in their patient, which they linked to the absence of utriculosaccular ducts in Ngn1-null mice. Malfunction of cranial nerve $V$ was assumed, but loss of sensation in this nerve's territory or its absence on brain MRI were not described. Based on the reported phenotype of Ngn1-null mutant mice, Schroder et al. proposed NEUROG1 as a new candidate gene for a congenital cranial dysinnervation disorder including cranial nerves $\mathrm{V}$ and $\mathrm{VIII}$, and excluded TIFAB and DCNP1 as causative. ${ }^{19-21}$

Finally, in 2015, Yavarna et al. identified a patient with corneal opacity, absent corneal reflexes and intellectual disability who had a homozygous missense variant in NEUROG1: c.347G > T, p. Arg116Leu. ${ }^{5}$ A summary of the clinical phenotypes of these patients is shown in Table 1.

Taken together, the very unusual combination of clinical features, and the striking similarity in findings between these three patients and the characteristics of the knock-out mouse model, provide compelling evidence that loss of NEUROG1 is causing CCDDs in humans. Our data strengthens the association of NEUROG1 with a very distinctive phenotype that comprises features related to hypoplasia/absence of the Vth and VIIIth cranial nerves, mild intellectual disability and, possibly, a wider clinical spectrum including manifestations such as

TAB LE 1 Comparison of clinical data between Schroder and Yavarnas's cases and our patient

\begin{tabular}{|c|c|c|c|}
\hline & Schroder et al., 2013 & Yavarna et al., 2015 & Our patient \\
\hline \multicolumn{4}{|l|}{ Neonatal period } \\
\hline Normal birth parameters & Yes & $\mathrm{nk}$ & Yes \\
\hline Poor sucking and swallowing & $\mathrm{nk}$ & $\mathrm{nk}$ & Yes \\
\hline Global hypotonia & Yes & $\mathrm{nk}$ & Yes \\
\hline Torticollis & Yes & $\mathrm{nk}$ & No \\
\hline \multicolumn{4}{|l|}{ Growth and development } \\
\hline Weight and height between 3rd-97th centiles & $\begin{array}{l}\text { Yes (weight 50th centile and } \\
\text { height } 5 \text { th centile) }\end{array}$ & $\mathrm{nk}$ & $\begin{array}{l}\text { Yes (weight } 3 \text { th centile and } \\
\text { height } 10 \text { th centile) }\end{array}$ \\
\hline Head circumference between 3 rd-97th centiles & Yes (75th centile) & $\mathrm{nk}$ & Yes (25th centile) \\
\hline $\mathrm{DD} / \mathrm{ID}$ & Yes (mild) & Yes (mild) & Yes (mild) \\
\hline \multicolumn{4}{|l|}{ Neurological involvement } \\
\hline Lagopthalmos, absent corneal reflex & $\mathrm{nk}$ & Yes & Yes \\
\hline Bilateral loss of facial sensation & $\mathrm{nk}$ & $\mathrm{nk}$ & Yes \\
\hline Bilateral sensorineural hearing loss & Yes (profound) & $\mathrm{nk}$ & Yes (profound) \\
\hline Oromotor dysfunction & $\begin{array}{l}\text { Yes (increased salivation, } \\
\text { inability to chew, mashed diet) }\end{array}$ & $\mathrm{nk}$ & $\begin{array}{l}\text { Yes (chewing/swallowing } \\
\text { difficulties, unable to close } \\
\text { the mouth) }\end{array}$ \\
\hline Balance disorder & Yes & $\mathrm{nk}$ & Yes (ataxic gait) \\
\hline Muscle weakness & Yes & nk & No \\
\hline Epilepsy & No & $\mathrm{nk}$ & $\begin{array}{l}\text { Yes (brief bilateral } \\
\text { myoclonic seizures) }\end{array}$ \\
\hline \multicolumn{4}{|l|}{ Craniofacial features } \\
\hline Cranial conformation & plagioscaphocephaly & $\mathrm{nk}$ & plagiocephaly \\
\hline Long face & Yes & $\mathrm{nk}$ & Yes \\
\hline Lack of facial mimicry & Yes & $\mathrm{nk}$ & Yes \\
\hline Facial asymmetry & Yes & $\mathrm{nk}$ & Yes \\
\hline High arched eyebrows and elongated palpebral & Yes & nk & Yes \\
\hline
\end{tabular}


TABLE 1 (Continued)

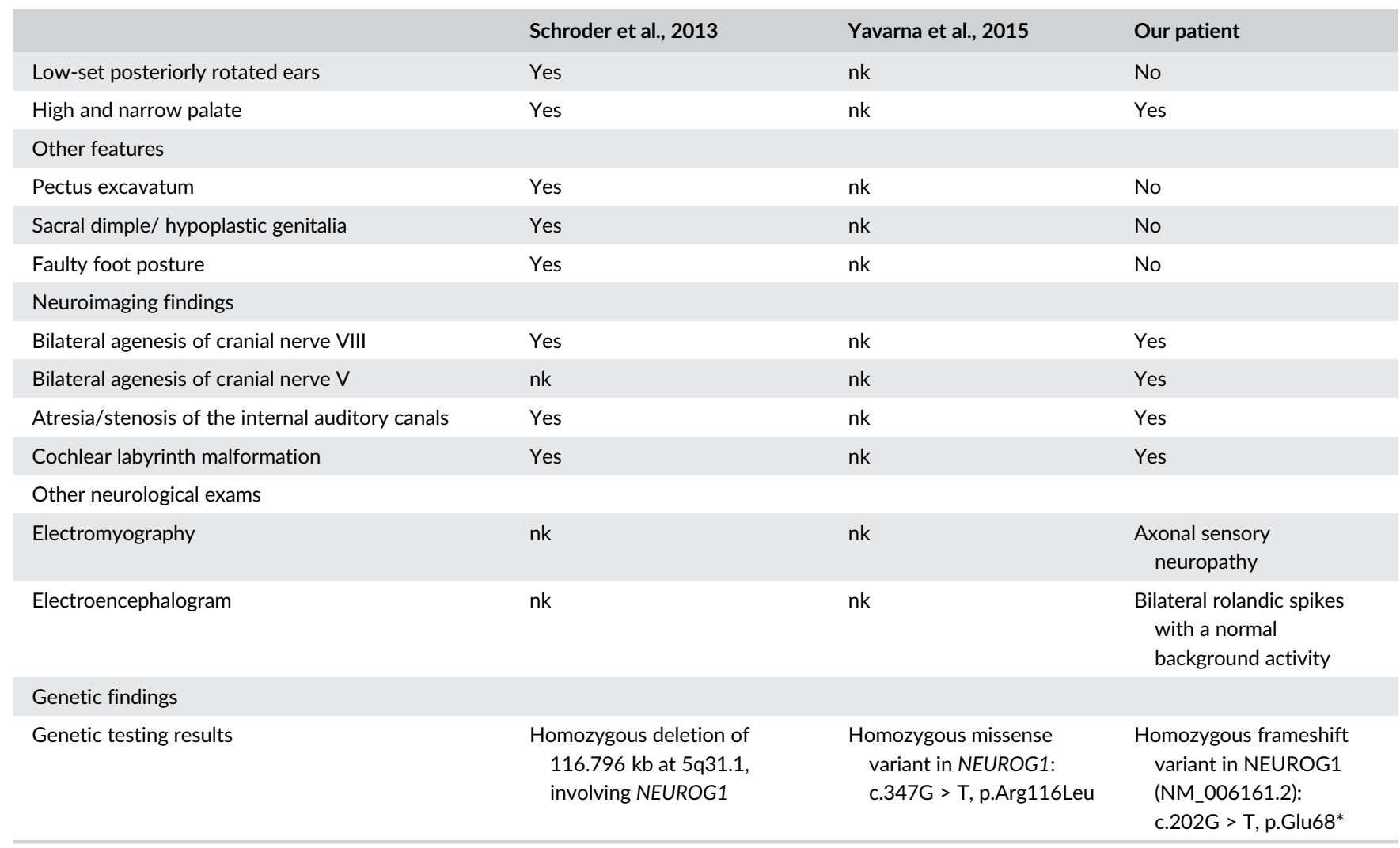

Abbreviations: DD/ID, developmental delay/intellectual disability; nk, not known.

epilepsy and axonal sensory neuropathy; and contributes to a better understanding and classification of these disorders.

\section{ACKNOWLEDGEMENTS}

We wish to thank the family that participated in our study.

\section{CONFLICT OF INTEREST}

The authors declare that they have no conflict of interest.

\section{PEER REVIEW}

The peer review history for this article is available at https://publons. com/publon/10.1111/cge.13922.

\section{DATA AVAILABILITY STATEMENT}

The data that support the findings of this study are available on request from the corresponding author. The data are not publicly available due to privacy or ethical restrictions.

\section{ORCID}

Juliette Dupont (D) https://orcid.org/0000-0001-8257-5067

Aida Maria Bertoli-Avella (D) https://orcid.org/0000-0001-9544-1877

\section{REFERENCES}

1. Gutowski NJ, Bosley TM, Engle EC. The congenital cranial dysinnervation disorders (CCDDs). Neuromuscul Disord. 2003;13: 573-578.
2. Takano-Maruyama M, Chen Y, Gaufo GO. Differential contribution of Neurog1 and Neurog2 on the formation of cranial ganglia along the anterior-posterior axis. Dev Dyn. 2012;241:229-241.

3. Sun Y, Nadal-Vicens M, Misono S, et al. Neurogenin promotes neurogenesis and inhibits glial differentiation by independent mechanisms. Cell. 2001;104:365-376.

4. Schröder JC, Läßig AK, Galetzka D, et al. A boy with homozygous microdeletion of NEUROG1 presents with a congenital cranial dysinnervation disorder [Moebius syndrome variant]. Behav Brain Funct. 2013;18(9):7.

5. Yavarna T, Al-Dewik N, Al-Mureikhi M, et al. High diagnostic yield of clinical exome sequencing in middle eastern patients with Mendelian disorders. Hum Genet. 2015;134:967-980.

6. Trujillano D, Bertoli-Avella AM, Kumar Kandaswamy K, et al. Clinical exome sequencing: results from 2819 samples reflecting 1000 families. Eur J Hum Genet. 2017;25:176-182.

7. Bauer P, Kandaswamy KK, Weiss MER, et al. Development of an evidence-based algorithm that optimizes sensitivity and specificity in ES-based diagnostics of a clinically heterogeneous patient population. Genet Med. 2019;21:53-61.

8. Trujillano D, Oprea GE, Schmitz Y, Bertoli-Avella AM, Abou Jamra R, Rolfs A. A comprehensive global genotype-phenotype database for rare diseases. Mol Genet Genomic Med. 2017;5:66-75.

9. Tomas-Roca L, Tsaalbi-Shtylik A, Jansen JG, et al. De novo mutations in PLXND1 and REV3L cause Möbius syndrome. Nat Commun. 2015;6:7199.

10. Miyake N, Chilton J, Psatha M, et al. Human CHN1 mutations hyperactivate $\alpha 2$-chimaerin and cause Duane's retraction syndrome. Science. 2008;321:839-843.

11. Park JG, Tischfield MA, Nugent AA, et al. Loss of MAFB function in humans and mice causes Duane syndrome, aberrant extraocular muscle innervation, and inner-ear defects. Am J Hum Genet. 2016;98: 1220-1227. 
12. Al-Baradie R, Yamada $\mathrm{K}, \mathrm{St}$ Hilaire $\mathrm{C}$, et al. Duane radial ray syndrome (Okihiro syndrome) maps to $20 \mathrm{q} 13$ and results from mutations in SALL4, a new member of the SAL family. Am J Hum Genet. 2002;71: 1195-1199.

13. Kohlhase J, Heinrich M, Schubert L, et al. Okihiro syndrome is caused by SALL4 mutations. Hum Mol Genet. 2002;11:29792987.

14. Kim EJ, Hori K, Wyckoff A, et al. Spatiotemporal fate map of neurogenin1 (Neurog1) lineages in the mouse central nervous system. J Comp Neurol. 2011;519:1355-1370.

15. Ma Q, Anderson DJ, Fritzsch B. Neurogenin 1 null mutant ears develop fewer, morphologically normal hair cells in smaller sensory epithelia devoid of innervation. J Assoc Res Otolaryngol. 2000;1: 129-143.

16. Ma Q, Chen Z, del Barco Barrantes I, et al. Neurogenin1 is essential for the determination of neuronal precursors for proximal cranial sensory ganglia. Neuron. 1998;20:469-482.

17. Han S, Dennis DJ, Balakrishnan A, et al. A non-canonical role for the proneural gene Neurog1 as a negative regulator of neocortical neurogenesis. Development. 2018;145(19):dev157719.

18. Vermeiren S, Bellefroid EJ, Desiderio S. Vertebrate sensory ganglia: common and divergent features of the transcriptional programs generating their functional specialization. Front Cell Dev Biol. 2020;8: 587-699.
19. Matsumura T, Kawamura-Tsuzuku J, Yamamoto T, Semba K, Inoue Jl. TRAF-interacting protein with a forkhead-associated domain B (TIFAB) is a negative regulator of the TRAF6-induced cellular functions. J Biochem. 2009;146:375-381.

20. Kim Y, Park CS, Shin HD, et al. A promoter nucleotide variant of the dendritic cell-specific DCNP1 associates with serum IgE levels specific for dust mite allergens among the Korean asthmatics. Genes Immun. 2007;8:369-378.

21. Zhou T, Wang S, Ren H, et al. Dendritic cell nuclear protein-1, a novel depression-related protein, upregulates corticotropin-releasing hormone expression. Brain. 2010;133:3069-3079.

\section{SUPPORTING INFORMATION}

Additional supporting information may be found online in the Supporting Information section at the end of this article.

How to cite this article: Dupont J, Vieira JP, Tavares ALT, et al. Adding evidence to the role of NEUROG1 in congenital cranial dysinnervation disorders. Clinical Genetics. 2021;99:

588-593. https://doi.org/10.1111/cge.13922 